\title{
Salt Preference and the Incidence of Cardiovascular Disease in a Japanese General Population: The Jichi Medical School Cohort Study
}

\author{
Saki Tadenuma1,2, Hideyuki Kanda1 ${ }^{*}$, Shizukiyo Ishikawa ${ }^{3}$, Kazunori Kayaba ${ }^{4}$, \\ Tadao Gotoh' ${ }^{5}$ Yosikazu Nakamura6, Eiji Kajii ${ }^{3}$ \\ ${ }^{1}$ Department of Environmental Health and Public Health, Faculty of Medicine, Shimane University, Shimane, \\ Japan \\ ${ }^{2}$ Department of Anesthesiology, Faculty of Medicine, Shimane University, Shimane, Japan \\ ${ }^{3}$ Division of Community and Family Medicine, Center for Community Medicine, Jichi Medical University, Tochigi, \\ Japan \\ ${ }^{4}$ Graduate School of Saitama Prefectural University, Saitama, Japan \\ ${ }^{5}$ Wara National Health Insurance Clinic, Gifu, Japan \\ ${ }^{6}$ Department of Public Health, Jichi Medical University, Tochigi, Japan \\ Email: *h-kanda@med.shimane-u.ac.jp
}

Received 17 December 2015; accepted 22 January 2016; published 25 January 2016

Copyright (C) 2016 by authors and Scientific Research Publishing Inc.

This work is licensed under the Creative Commons Attribution International License (CC BY).

http://creativecommons.org/licenses/by/4.0/

(c) (i) Open Access

\section{Abstract}

Dietary salt intake has been reported to be associated with cardiovascular disease (CVD). However, there were few studies that assessed the relationship of salt preference with CVD. We examined the association between salt preference and the incidence of CVD and its subtypes in a Japanese general population. Based on the prospective Jichi Medical School Cohort Study, data were analyzed from 11,394 eligible participants. A baseline survey of the preference for salt was obtained by questionnaire and health examinations from April 1992 through July 1995 in 12 communities in Japan. The participants were followed up until December 2005 (mean follow-up period, $10.7 \pm$ 2.4 years). Subjects were divided into three categories according to their preference for salt: favor, so-so, and disfavor. A Cox proportional hazards model was used to calculate hazard ratios (HRs) of the incidence of CVD according to the preference categories. We observed 485 cardiovascular events (258 in men and 227 in women). Among the men, the multivariable adjusted HRs for incidence of myocardial infarction and subarachnoid hemorrhage for favor versus so-so salt preference were $0.34(95 \%$ confidence interval, $0.17-0.71)$ and $7.10(0.88-56.84)$, respectively.

"Corresponding author.

How to cite this paper: Tadenuma, S., Kanda, H., Ishikawa, S., Kayaba, K., Gotoh, T., Nakamura, Y. and Kajii, E. (2016) Salt Preference and the Incidence of Cardiovascular Disease in a Japanese General Population: The Jichi Medical School Cohort Study. Health, 8, 105-115. http://dx.doi.org/10.4236/health.2016.81013 
Among the women, age-adjusted HRs for the incidence of CVD, total stroke, cerebral hemorrhage, and cerebral infarction for the favor preference were $1.41(1.02-1.95), 1.36(0.97-1.91), 1.79$ $(0.87-3.71)$, and $1.40(0.89-2.19)$, respectively. The data indicated that preference for salt may be associated with an increase in the incidence of CVD in women.

\section{Keywords}

\section{Salt Preference, Cardiovascular Disease, Cohort Study, Japanese}

\section{Introduction}

Cardiovascular diseases (CVD), such as coronary heart disease (CHD) and stroke, are common causes of death and disabilities for elders in developed countries, including Japan, after hypertension and atherosclerosis. An estimated 17.5 million people died from CVD in 2012 (31\% of all global deaths). Tobacco use, unhealthy diet and obesity, physical inactivity and harmful use of alcohol, diabetes and hyperlipidemia, hypertension and atherosclerosis are established risk factors for CVD. One of main causes on hypertension is much more salt intakes. Salt intakes influence individual salt preferences strongly [1].

Excessive salt intake affects the incidence and prevalence of hypertension, and subsequently influences the prevalence of cardiovascular disease (CVD) [2]. High salt intake has also been associated with increased CVD mortality and incidence [3]-[6]. The Japanese are known to have higher salt intake than many other populations [7]. In Japan, the mean salt intake among adults was $10.2 \mathrm{~g}$ per day (men, $11.3 \mathrm{~g}$ per day; women, $9.4 \mathrm{~g}$ per day) according to a national nutrition survey in 2013 [8]. Now, a new goal has been set to improve the level of salt intake among Japanese to within 8 g per day [9]. Therefore, dietary sodium restriction must be recommended to a considerable number of people. It is important to estimate salt intake and advice participants who consume excessive amounts of salt to reduce their salt intake. In general, daily salt intake may be estimated by a food frequency questionnaire or by measurement of 24 hour urinary sodium excretion [10]. However, both methods seem inconvenient for general use in mass screening. For these reasons, at health check-up centers or outpatient clinics, salt intake is usually estimated by a questionnaire on salt preference [11] [12].

Salt preference is thought to be associated with salt intake [13]. In a prospective study that examined the relationship between salt preference and CVD, salt preference was significantly positively associated with dietary sodium intake. Compared to the low salt preference group, the high salt preference group showed a relation to higher mortality from stroke [11]. However, few researches have attempted to assess the effects of salt preference on CVD. We could find no studies that clarified the relationships between salt preference and mortality from subtypes of CVD. As far as we know, no previous studies have reported an association of salt preference with the incidence of CVD and its subtypes.

Therefore, the aim of this study was to clarify the relationships between salt preference and the incidence of CVD and CVD subtypes using about 10 years of follow-up data from a large-scale prospective population-based cohort study conducted in Japan.

\section{Subjects and Methods}

\subsection{Subjects}

The Jichi Medical School (JMS) Cohort Study is a population-based prospective study that was started in 1992 to investigate the risk factors for CVD in 12 rural areas in Japan. A total of 12,490 people (4911 men and 7579 women) were enrolled in this study. Mass screening examinations for CVD have been conducted in Japan since 1982 under the direction of the Health and Medical Service Law for the Aged, and we used this system to collect the data. The baseline data were obtained from April 1992 through July 1995. Baseline examinations consisted of physical and blood examinations and a self-administered questionnaire. A detailed description of the standardized collection of baseline examinations was published previously [14].

Among the 12,490 participants, 95 (0.8\%) declined follow-up and 7 (0.06\%) could not be contacted after baseline examination, after which 12,388 subjects (4869 men and 7519 women) remained. We excluded partic- 
ipants with a history of CVD (96 men and 74 women) and those with missing data on salt preference (356 men and 468 women). Ultimately, 11,394 subjects (4417 men and 6977 women) were analyzed in the present study. Written informed consent to participate in the study was obtained individually from all of the participants in the mass screening. This study was approved by the Institutional Review Board of Jichi Medical School.

\subsection{Baseline Examination}

The health checkup was carried out in all 12 communities using same protocols. The body height of all participants was measured without shoes. Body weight while fully clothed was recorded; $0.5 \mathrm{~kg}$ in summer or $1 \mathrm{~kg}$ in other seasons was subtracted from the recorded weight. Body mass index (BMI) was calculated as weight (kg)/ height $(\mathrm{m})^{2}$. Systolic blood pressure (SBP) and diastolic blood pressure (DBP) at baseline were measured with a fully automated sphygmomanometer (BP203RV-II; Nippon Colin, Komaki, Japan), which was placed on the right arm of the participant after resting for at least 5 minutes in a sitting position. Serum cholesterol concentration was measured by taking a blood sample from the antecubital vein of the seated participants. Total cholesterol was measured using an enzymatic method, and high density lipoprotein cholesterol (HDL-C) was measured using the phosphotungstate precipitation method (Wako, Osaka, Japan; inter-assay coefficient of variation, $1.5 \%)$.

Information on age, lifestyle, and medical history was obtained from responses to the baseline questionnaire. Salt preference was ascertained with the following question: "Do you like salty foods?" Participants answered with 1 of 5 multiple choice options: "highly favor”, “favor”, "so-so”, “moderately disfavor”, or "disfavor”. Subjects were divided into three categories of salt preference according to their response: favor: "highly favor" or "moderately favor"; so-so: "so-so"; and disfavor: "moderately disfavor" or "disfavor".

Smoking habit and alcohol drinking habit were determined from the baseline questions on current smoking and current drinking. Histories of hypertension, diabetes, and hyperlipidemia were determined from questions on the medical history of each illness. Response to the number of years of education was in terms of consecutive years; the response was then categorized as $\geq 9$ years or $<9$ years.

\subsection{Follow-Up}

The national mass screening system used to obtain the baseline data for the JMS Cohort Study was also used to follow the subjects each year. Subjects were asked whether they had a history of CVD after enrolling. Follow-up was conducted from 1995 to 2005 . The mean follow-up period \pm standard deviation (SD) was $10.7 \pm 2.4$ years. Subjects who did not attend a follow-up examination were contacted by mail or telephone. If an incident case of stroke or myocardial infarction (MI) was suspected, those subjects with such histories were asked when and which hospital they visited. Medical records pertaining to stroke and MI were checked if the subjects were hospitalized for any reason, and incident cases were recorded. If both MI and stroke had occurred during the follow-up period, each of the endpoints of stroke and MI was counted as the first for each disease. The CVD endpoint was defined as stroke or MI, whichever occurred first. Death from CVD was also included in the CVD incidence data. Information on death was obtained from death certificates, which were collected at public health centers with the official permission from the Japanese Ministry of General Affairs and the Ministry of Health, Labour and Welfare until the end of 2005. Data on subjects who moved out of the study area during the follow-up period were obtained annually from the municipal government.

\subsection{Diagnostic Criteria}

If a CVD event was suspected, we requested duplicate images from computed tomography or magnetic resonance imaging (in cases of stroke) or electrocardiograms (in cases of MI). The diagnoses were determined independently by a diagnosis committee in the JMS Cohort Study Group composed of a radiologist, a neurologist, and two cardiologists. Criteria for stroke were a focal and nonconvulsive neurological deficit of sudden onset persisting longer than 24 hours. Stroke subtypes were categorized as cerebral hemorrhage, cerebral infarction, or subarachnoid hemorrhage (SAH) according to the criteria of the National Institute of Neurological Disorder and Stroke [15]. MI was diagnosed according to the criteria of the World Health Organization Multinational Monitoring of Trends and Determinants in Cardiovascular Disease (MONICA) Project [16]. 


\subsection{Statistical Analysis}

All analyses were conducted according to subject gender. Descriptive parameters are shown as the mean, standard deviation, or proportion (\%). We compared characteristics between salt preference groups by the chi-square test or one-way analysis of variance. Finally, Cox proportional hazards models were used to calculate hazard ratios (HRs) with 95\% confidence intervals (CIs) for the incidence of CVD according to salt preference, after adjusting for age, smoking habit, alcohol drinking habit, history of hyperlipidemia, and years of education (HR-all*) for men, and after adjusting for age, smoking habit, and alcohol drinking habit, BMI, HDL-C, and years of education (HR-all ${ }^{\dagger}$ ) for women, which were considered to be potential confounding factors. HRs of each incidence of stroke, stroke subtypes, and MI were calculated by same statistical models. All p values were two-tailed, and a probability value $<0.05$ was considered statistically significant. All analyses were performed using the Statistical Package for Social Science (SPSS) for Windows, version 16.0 (SPSS Inc., Japan).

\section{Results}

During a mean follow-up period of 10.7 years, we documented 485 CVD events (258 in men, 227 in women): 415 strokes (210 in men, 205 in women), including 264 cerebral infarctions (150 in men, 114 in women), 94 hemorrhagic strokes (47 in men, 47 in women), and 56 SAHs (13 in men, 43 in women), and 76 MIs (52 in men, 24 in women).

The baseline characteristics of the subjects by salt preference group are shown in Table 1 . In both men and women, favor salt preference was positively associated with smoking ( $<<0.01$ for men; $p=0.01$ for women) and alcohol drinking ( $<0.01$ for men; $p=0.02$ for women). Among the men, those in the favor salt preference group tended to be younger, more highly educated (both, $\mathrm{p}<0.01)$, and less likely to have hyperlipidemia $(\mathrm{p}=$ 0.04). Among the women, those in the favor salt preference group tended to be older, less well educated (both, $p$ $<0.01)$ and more likely to have both a higher incidence of CVD $(p=0.046)$ and higher BMI $(p<0.01)$ and a lower serum concentration of HDL-C $(\mathrm{p}<0.01)$.

The incidence and HRs for CVD by salt preference category are shown in Table 2. After adjustment for age, there were no significant associations between salt preference and CVD or total stroke among the men. Our data showed 11 MIs, and the HR for MI was significantly lower in the favor salt subjects compared with so-so subjects (HR, 0.34; 95\% CI, 0.17 - 0.68). After further multiple adjustment for smoking status, alcohol drinking status, history of hyperlipidemia, and years of education, the HR for MI was 0.35 (0.17 - 0.71). Among women, the HR for CVD was significantly higher in the favor salt subjects compared with the so-so subjects (HR, 1.41; 95\% CI, 1.02 - 1.95) after adjustment for age. After further adjustment for smoking status, alcohol drinking status, BMI, HDL-C, and years of education, the HR was 1.15 (0.81 - 1.63). The HR for total stroke was also high (1.36; 95\% CI, 0.97 - 1.91) in the favor salt subjects among women. After multivariate adjustment (HR-all $\left.{ }^{\dagger}\right)$, HR was 1.08 (0.74 - 1.57). No significant association was found between salt preference and MI in the women, although a significant association was found in the men.

We also analyzed the respective association between salt preference and the incidences of stroke subtypes (Table 3). Among the men, there were 9 SAHs, and the HR was $8.09(1.02$ - 63.84) in the favor salt subjects after adjustment for age. After multivariate adjustment (HR-all $\left.{ }^{*}\right)$, the HR of SAHs was 7.10 (0.88 - 56.84). Among the women, age-adjusted HRs for cerebral hemorrhage and cerebral infarction were $1.79(0.87-3.71)$ and 1.40 (0.89 - 2.19), respectively, in the favor salt subjects. After multivariate adjustment (HR-all $\dagger$ ), HRs were 1.59 (0.74 - 3.44) and 1.07 (0.65 - 1.78), respectively.

\section{Discussion}

We investigated the association between salt preference and the incidence of CVD in a Japanese general population. We found that salt preference was positively associated with an increased risk of SAH and a decreased risk of MI in men. For women, salt preference was positively associated with an incidence of CVD after age-adjustment. HRs for incidences of cerebral hemorrhage and cerebral infarction were also higher, although not with significance. To our knowledge, this study is the first prospective study to provide evidence of the relationship of salt preference with the incidence of stroke.

We found that salt preference was associated with an increased incidence of CVD in the women. We examined CVD incidence data rather than mortality data as endpoints. Because the incidence of CVD occurs earlier 
Table 1. Baseline characteristics of study participants by salt preference categories.

\begin{tabular}{|c|c|c|c|c|}
\hline & Favor & So-so & Disfavor & \\
\hline (Men) number (n) & 1813 & 1585 & 1019 & $\mathrm{p}$-value ${ }^{\mathrm{a}}$ \\
\hline Number of CVD incidence & $91(5.0)$ & $102(6.4)$ & $65(6.4)$ & 0.15 \\
\hline Number of Stroke incidence & $81(4.5)$ & $76(4.8)$ & $53(5.2)$ & 0.68 \\
\hline \multirow[t]{2}{*}{ Number of MI incidence } & $11(0.6)$ & $29(1.8)$ & $12(1.2)$ & $<0.01^{* *}$ \\
\hline & & & & $\mathrm{p}$-value ${ }^{\mathrm{b}}$ \\
\hline Age (year) & $54.5(11.5)$ & $54.6(12.1)$ & $56.7(12.4)$ & $<0.01^{* *}$ \\
\hline Systolic blood pressure (mmHg) & $131.6(20.6)$ & $131.1(21.0)$ & $131.0(21.0)$ & 0.66 \\
\hline Diastolic blood pressure (mmHg) & $79.3(12.3)$ & $79.0(12.1)$ & $79.0(12.6)$ & 0.71 \\
\hline Total-cholesterol (mg/dl) & $183.8(34.4)$ & $186.3(33.7)$ & $185.3(34.7)$ & 0.10 \\
\hline HDL-cholesterol (mg/dl) & $49.3(13.6)$ & $49.0(13.6)$ & $48.1(13.0)$ & 0.08 \\
\hline Body mass index $\left(\mathrm{kg} / \mathrm{m}^{2}\right)$ & $23.0(2.9)$ & $23.0(2.9)$ & $22.9(2.9)$ & 0.51 \\
\hline Current Smokers & 55.8 & 49.2 & 43.4 & $<0.01^{* *}$ \\
\hline Current Drinkers & 78.2 & 75.5 & 70.4 & $<0.01^{* *}$ \\
\hline History of hypertension & 8.8 & 9.8 & 10.7 & 0.27 \\
\hline History of diabetes mellitus & 1.9 & 2.7 & 3.0 & 0.12 \\
\hline History of hyperlipidemia & 0.7 & 1.4 & 1.8 & $0.04^{*}$ \\
\hline Education years (over 9 years) & 88.5 & 88.7 & 83.4 & $<0.01^{* *}$ \\
\hline (Women) number (n) & 1618 & 2885 & 2474 & $\mathrm{p}$-value ${ }^{\mathrm{a}}$ \\
\hline Number of CVD incidence & $67(4.1)$ & $80(2.8)$ & $80(3.2)$ & $0.046^{*}$ \\
\hline Number of stroke incidence & $60(3.7)$ & $74(2.6)$ & $71(2.9)$ & 0.09 \\
\hline \multirow[t]{2}{*}{ Number of MI incidence } & $7(0.4)$ & $8(0.2)$ & $9(0.4)$ & 0.68 \\
\hline & & & & $\mathrm{p}$-value ${ }^{\mathrm{b}}$ \\
\hline Age (year) & $55.8(11.0)$ & $54.8(11.0)$ & $55.5(11.5)$ & $<0.01^{*}$ \\
\hline Systolic blood pressure (mmHg) & $128.9(21.5)$ & $127.9(20.9)$ & $127.6(21.0)$ & 0.15 \\
\hline Diastolic blood pressure (mmHg) & $76.8(12.3)$ & $76.2(12.0)$ & $76.0(12.1)$ & 0.08 \\
\hline Total-cholesterol (mg/dl) & $196.5(34.1)$ & $197.2(35.5)$ & $197.1(34.5)$ & 0.81 \\
\hline HDL-cholesterol (mg/dl) & $51.5(12.0)$ & $52.5(12.5)$ & $53.8(12.7)$ & $<0.01^{* *}$ \\
\hline Body Mass Index $\left(\mathrm{kg} / \mathrm{m}^{2}\right)$ & $23.5(3.3)$ & $23.1(3.1)$ & $23.0(3.2)$ & $<0.01^{* *}$ \\
\hline Current smokers & 6.9 & 5.1 & 5.0 & $0.01^{*}$ \\
\hline Current drinkers & 26.9 & 25.6 & 23 & $0.02^{*}$ \\
\hline History of hypertension & 12.9 & 11.6 & 12.4 & 0.39 \\
\hline History of diabetes mellitus & 1.2 & 1.6 & 2.0 & 0.14 \\
\hline History of hyperlipidemia & 1.5 & 2.3 & 2.1 & 0.16 \\
\hline Education years (over 9 years) & 75.9 & 81.2 & 80.0 & $<0.01^{* *}$ \\
\hline
\end{tabular}

Data are expressed as a mean (standard deviation) for variables or as a percentage of the population. ${ }^{\mathrm{a}}$ Chi-square test; ${ }^{\mathrm{b}}$ Analysis of variance (ANOVA); ${ }^{*}$ p values were $<0.05 ;{ }^{* *}$ p values were $<0.01$. 
Table 2. Hazard ratios and 95\% CIs of incidence from cardiovascular disease and myocardial infarction with gender difference by salt preference categories.

\begin{tabular}{|crcr}
\hline \multicolumn{2}{c}{ Salt preference category } \\
\hline \\
\hline Fen & Favor & So-so & Disfavor \\
\hline Men & & \\
\hline
\end{tabular}

Cardiovascular disease $^{\S}$

$\mathrm{N}$

HR-age

HR-all ${ }^{*}$

Total-stroke

$\mathrm{N}$

HR-age

HR-all*

Myocardial infarction

N

HR-age

HR-all*

Women

Cardiovascular disease $\mathrm{e}^{\S}$

N

HR-age

HR-all ${ }^{\dagger}$

Total-stroke

$\mathrm{N}$

HR-age

HR-all ${ }^{\dagger}$

Myocardial infarction

$$
\mathrm{N}
$$

HR-age

HR-all ${ }^{\dagger}$
91

0.80 (0.60 - 1.06)

$0.75(0.57-1.01)$

81

0.97 (0.71 - 1.33)

0.90 (0.64 - 1.25)

11

0.34 (0.17 - 0.68)

0.35 (0.17 - 0.71)

67

1.41 (1.02 - 1.95)

1.15 (0.81 - 1.63)

60

1.36 (0.97 - 1.91)

$1.08(0.74-1.57)$

7

1.44 (0.52 - 3.98)

1.37 (0.49 - 3.82)
102

1.00

1.00

76

1.00

1.00

29

1.00

1.00

65

$0.83(0.61$ - 1.13)

$0.84(0.61-1.17)$

53

0.9 (0.63 - 1.28)

$0.89(0.62-1.28)$

12

$0.55(0.28$ - 1.09)

0.64 (0.33 - 1.24)

HR-age: hazard ratios adjusted for age. HR-all': hazard ratios adjusted for age, smoking status and drinking status, history of hyperlipidemia, and education years. HR-all ${ }^{\dagger}$ : hazard ratios adjusted for age, smoking status and drinking status, BMI, HDL-choesterol, and education years. ${ }^{\S}$ : The case which occurred both stroke and myocardial infarction is included.

than that of mortality, our study was significant in that we captured the risk of CVD at an earlier stage. With respect to Japanese studies, the Japan Collaborative Cohort Study for Evaluation of Cancer Risk (JACC Study) reported an association between salt preference and mortality from stroke among Japanese men and women [11]. In that study, subjects were divided into three categories according to their preference answer. Compared to the low salt preference group, the salt preference group was associated with higher mortality from stroke after 16.4 years of follow-up: The multivariable HRs for CVD were $1.05(0.92$ - 1.20) for men and $1.05(0.92$ - 1.19) for 
Table 3. Hazard ratios and 95\%CIs of incidence of subtypes of stroke with gender differrence by salt preference categories.

\begin{tabular}{|c|c|c|c|}
\hline \multicolumn{4}{|c|}{ Salt preference category } \\
\hline & Favor & So-so & Disfavor \\
\hline \multicolumn{4}{|l|}{ Men } \\
\hline \multicolumn{4}{|c|}{ Cerebral hemorrhage } \\
\hline $\mathrm{N}$ & 18 & 17 & 12 \\
\hline HR-age & $0.96(0.49-1.87)$ & 1.00 & $0.92(0.44-1.91)$ \\
\hline HR-all ${ }^{*}$ & $0.84(0.41-1.70)$ & 1.00 & $0.86(0.40-1.88)$ \\
\hline \multicolumn{4}{|c|}{ Cerebral infarction } \\
\hline $\mathrm{N}$ & 54 & 58 & 38 \\
\hline HR-age & $0.85(0.59-1.24)$ & 1.00 & $0.84(0.56-1.26)$ \\
\hline HR-all ${ }^{*}$ & $0.80(0.54-1.17)$ & 1.00 & $0.86(0.56-1.32)$ \\
\hline \multicolumn{4}{|c|}{ Subarachnoid hemorrhage } \\
\hline $\mathrm{N}$ & 9 & 1 & 3 \\
\hline HR-age & $8.09(1.02-63.84)$ & 1.00 & $4.12(0.42-39.78)$ \\
\hline HR-all ${ }^{*}$ & $7.10(0.88-56.84)$ & 1.00 & $2.62(0.24-29.10)$ \\
\hline \multicolumn{4}{|l|}{ Women } \\
\hline \multicolumn{4}{|c|}{ Cerebral hemorrhage } \\
\hline $\mathrm{N}$ & 15 & 14 & 18 \\
\hline HR-age & $1.79(0.87-3.71)$ & 1.00 & $1.42(0.70-2.85)$ \\
\hline HR-all ${ }^{\dagger}$ & $1.59(0.74-3.44)$ & 1.00 & $1.59(0.78-3.27)$ \\
\hline \multicolumn{4}{|c|}{ Cerebral infarction } \\
\hline $\mathrm{N}$ & 36 & 42 & 36 \\
\hline HR-age & $1.40(0.89-2.19)$ & 1.00 & $0.93(0.59-1.45)$ \\
\hline HR-all ${ }^{\dagger}$ & $1.07(0.65-1.78)$ & 1.00 & $0.94(0.59-1.49)$ \\
\hline \multicolumn{4}{|c|}{ Subarachnoid hemorrhage } \\
\hline $\mathrm{N}$ & 8 & 18 & 17 \\
\hline HR-age & $0.78(0.34-1.81)$ & 1.00 & $1.10(0.56-2.14)$ \\
\hline HR-all ${ }^{\dagger}$ & $0.65(0.27-1.56)$ & 1.00 & $1.05(0.54-2.07)$ \\
\hline
\end{tabular}

HR-age: hazard ratios adjusted for age. HR-all': hazard ratios adjusted for age, smoking status and drinking status, history of hyperlipidemia, and education years. HR-all ${ }^{\dagger}$ h hazard ratios adjusted for age, smoking status and drinking status, MI, and HDL-cholesterol, and education years.

women. HRs for stroke were $1.21(0.99$ - 1.49) for men and $1.22(1.00$ - 1.49) for women. It is possible that the discrepancies in the results between the JACC Study and our study were due to differences in the number of participants, follow-up period, and examination end points. Our study suggested the possibility that salt preference was associated with the stage of CVD incidence rather than mortality, with gender differences.

High salt preference may result in a long-term, high-sodium intake, then leading to high blood pressure and an increased risk of CVD. Several previous studies reported positive associations between salt preference and salt intake [12] [17] [18] and between salt intake and the incidence of CVD or mortality [3]-[6]. Several explanations for the associations between sodium intake and CVD have been put forward. High salt intakes influence 
CVD by altering left ventricular mass [19] or increasing blood flow and vascular reactivity [20] [21]. In our study, the incidence of CVD was high for women. Our targets among women were almost local residents. So, the incidence of CVD may be slightly higher than the previous study. For women, high salt preference tended to be less well educated. Therefore, subjects with high salt preference may have behavioral risk factors, leading to higher risk of CVD in the women. Accordingly, women with a high salt preference may intake much more salt than those with a low salt preference. Further investigations are necessary to clarify sex difference in cardiovascular risk factors.

In our study, favor salt preference was positively associated with smoking and alcohol drinking in both men and women. The men in the favor salt preference group tended to be younger and more highly educated, whereas the women in the salt preference group tended to be older, less well educated, and more likely to have a higher BMI and lower serum concentration of HDL-C. Despite these results, salt preference was not associated with CVD risk factors such as SBP, DBP, and a history of hypertension. Our results suggest that salt preference may be one of the risk factors of premature CVD. It is also possible that some people with a high salt preference developed hypertension that led to CVD during the follow-up period. Among the women, salt preference was associated with the incidence of CVD after age adjustment. After adjustment for smoking status, alcohol drinking status, BMI, HDL-C, and years of education, the risk of CVD was attenuated. Accordingly, the influence of common risk factors on CVD incidence was strong for women, and salt preference may reflect accumulation of confounding risk factors except for age. These common factors can be self-managed and self-controlled. People indicating a high salt preference in the detailed interview during health check-ups should be recommended to practice adequate health behavior for the prevention of CVD in daily life, especially for women.

Salt preference and food intake are affected by socioeconomic and psychophysiological factors such as recent dietary habit, culture, and income [22] [23]. Taste preferences are acquired early in life through the process of choosing foods and actual salt intake [24]. Lampure et al. reported that as a new pathway without a common route that leads to CVD, salt preference was associated with uncontrolled eating behavior [25], which is the tendency to lose control over eating when hungry or when exposed to external stimuli. A previous study showed that binge eating was associated with a higher incidence of a new diagnosis of dyslipidemia, any metabolic syndrome component (hypertension, dyslipidemia, or type 2 diabetes), and two or more components of metabolic syndrome after 5 years of follow-up [26]. Thus, eating disorders such as uncontrolled eating behavior can result in a relationship between salt preference and the occurrence of CVD.

In our study, salt preference tended to be inversely associated with the incidence of MI in the men. Our results are similar to those of a previous study [11] although the reason for this cannot be fully explained. The decreased risk of MI associated with high salt preference might reflect the beneficial cardiovascular effects of the intake of $n-3$ polyunsaturated fatty acids and isoflavones in the inhibition of platelet aggregation, lowering of blood pressure, and modulation of the inflammatory system [27] [28]. For the men in the present study, the low incidence of MI and the high incidence of SAH were based on a small number of incident cases. Thus, there was wide range of $95 \%$ CIs for the point estimates.

For women, salt preference may reflect common risk factors that will cause CVD in the future and other unknown factors such as eating behavior in an earlier stage of life. Especially in women, early assessment of salt preference may be effective in reducing the incidence of CVD. For subjects with high salt preference, early intervention may be able to prevent excessive salt intake and uncontrolled eating behavior in the future.

Our study has several strengths. First, it was conducted as a large-scale multicenter cohort study. In addition, we investigated the incidence of not only CVD but also stroke subtypes as endpoints. However, there were several limitations. First, the follow-up period was shorter, and second, the numbers of subjects with an incidence of CVD were less than those of a previous study [11]. Third, estimated salt intake is not measured quantitatively. It is unclear whether the salt preference questionnaire reflects the responder's actual salt intake. Fourth, the participants were recruited through a mass screening program, and therefore, their concern for health may exceed that of the general population, which could result in differences in salt intake and other behavioral profiles. Finally, we were unable to control for other potential confounders such as nutrient factors and income.

\section{Conclusion}

We found that salt preference was positively associated with an increased risk of the incidence of SAH in men after multivariate adjustment and in CVD in women after adjustment for age. As with other common risk factors 
for CVD, assessing salt preference may lead to the prevention of CVD because such assessment may help to prevent excessive salt intake and uncontrolled eating behavior in the future. These tendencies may apply especially to women.

\section{Acknowledgements}

We are grateful to Mr. Yasuyuki Fujita and Mr. Tsuyoshi for the advice to this study. This study was supported by Grant-in-Aid from the Ministry of Education, Culture, Sports, Science and Technology, Japan, and grants from the Foundation for the Development of the Community, Tochigi, Japan.

\section{References}

[1] World Health Organization (2015) Cardiovascular Diseases. http://www.who.int/mediacentre/factsheets/fs317/en/

[2] He, F.J. and MacGregor, G.A. (2007) Salt, Blood Pressure and Cardiovascular Disease. Current Opinion in Cardiology, 22, 298-305. http://dx.doi.org/10.1097/HCO.0b013e32814f1d8c

[3] Gardener, H., Rundek, T., Wright, C.B., Elkind, M.S. and Sacco, R.L. (2012) Dietary Sodium and Risk of Stroke in the Northern Manhattan Study. Stroke, 43, 1200-1205. http://dx.doi.org/10.1161/STROKEAHA.111.641043

[4] Strazzullo, P., D’Elia, L., Kandala, N.B. and Cappuccio, F.P. (2009) Salt Intake, Stroke, and Cardiovascular Disease: Meta-Analysis of Prospective Studies. BMJ, 339, b4567. http://dx.doi.org/10.1136/bmj.b4567

[5] Tuomilehto, J., Jousilahti, P., Rastenyte, D., Moltchanov, V., Tanskanen, A., Pietinen, P. and Nissinen, A. (2001) Urinary Sodium Excretion and Cardiovascular Mortality in Finland: A Prospective Study. Lancet, 357, 848-851. http://dx.doi.org/10.1016/S0140-6736(00)04199-4

[6] Umesawa, M., Iso, H., Date, C., Yamamoto, A., Toyoshima, H., Watanabe, Y., Kikuchi, S., Koizumi, A., Kondo, T., Inaba, Y., Tanabe, N., Tamakoshi, A. and Group, J.S. (2008) Relations between Dietary Sodium and Potassium Intakes and Mortality from Cardiovascular Disease: The Japan Collaborative Cohort Study for Evaluation of Cancer Risks. The American Journal of Clinical Nutrition, 88, 195-202.

[7] Zhou, B.F., Stamler, J., Dennis, B., Moag-Stahlberg, A., Okuda, N., Robertson, C., Zhao, L., Chan, Q., Elliott, P. and Group, I.R. (2003) Nutrient Intakes of Middle-Aged Men and Women in China, Japan, United Kingdom, and United States in the Late 1990s: The INTERMAP Study. Journal of Human Hypertension, 17, 623-630. http://dx.doi.org/10.1038/sj.jhh.1001605

[8] Ministry of Health, of Health, Labour and Welfare (2013) National Health and Nutrition Survey Japan. (In Japanese). http://www.mhlw.go.jp/bunya/kenkou/eiyou/dl/h25-houkoku-04.pdf

[9] Ministry of Health Labour and Welfare (2012) The Basic Policies for Comprehensive Public Health Promotion (Health Japan 21, 2nd Term). http://www.mhlw.go.jp/file/06-Seisakujouhou-10900000-Kenkoukyoku/0000047330.pdf

[10] Bentley, B. (2006) A Review of Methods to Measure Dietary Sodium Intake. The Journal of Cardiovascular Nursing, 21, 63-67. http://dx.doi.org/10.1097/00005082-200601000-00012

[11] Ikehara, S., Iso, H., Date, C., Kikuchi, S., Watanabe, Y., Inaba, Y., Tamakoshi, A. and Group, J.S. (2012) Salt Preference and Mortality from Stroke and Coronary Heart Disease for Japanese Men and Women: The JACC Study. Preventive Medicine, 54, 32-37. http://dx.doi.org/10.1016/j.ypmed.2011.10.013

[12] Takamura, K., Okayama, M., Takeshima, T., Fujiwara, S., Harada, M., Murakami, J., Eto, M. and Kajii, E. (2014) Influence of Salty Food Preference on Daily Salt Intake in Primary Care. International Journal of General Medicine, 7, 205-210.

[13] Van der Veen, J.E., De Graaf, C., Van Dis, S.J. and Van Staveren, W.A. (1999) Determinants of Salt Use in Cooked Meals in The Netherlands: Attitudes and Practices of Food Preparers. European Journal of Clinical Nutrition, 53, 388394. http://dx.doi.org/10.1038/sj.ejcn.1600737

[14] Ishikawa, S., Gotoh, T., Nago, N. and Kayaba, K. (2002) The Jichi Medical School (JMS) Cohort Study: Design, Baseline Data and Standardized Mortality Ratios. Journal of Epidemiology/Japan Epidemiological Association, 12, 408417. http://dx.doi.org/10.2188/jea.12.408

[15] Adams Jr., H.P., Bendixen, B.H., Kappelle, L.J., Biller, J., Love, B.B., Gordon, D.L. and Marsh 3rd., E.E. (1993) Classification of Subtype of Acute Ischemic Stroke. Definitions for Use in a Multicenter Clinical Trial. TOAST. Trial of Org 10172 in Acute Stroke Treatment. Stroke, 24, 35-41. http://dx.doi.org/10.1161/01.STR.24.1.35

[16] World Health Organization (1988) The World Health Organization MONICA Project (Monitoring Trends and Determinants in Cardiovascular Disease): A Major International Collaboration. WHO MONICA Project Principal Investigators. Journal of Clinical Epidemiology, 41, 105-114. http://dx.doi.org/10.1016/0895-4356(88)90084-4

[17] Hashimoto, T., Yagami, F., Owada, M., Sugawara, T. and Kawamura, M. (2008) Salt Preference According to a Ques- 
tionnaire vs. Dietary Salt Intake Estimated by a Spot Urine Method in Participants at a Health Check-Up Center. Internal medicine, 47, 399-403. http://dx.doi.org/10.2169/internalmedicine.47.0622

[18] Takachi, R., Ishihara, J., Iwasaki, M., Ishii, Y. and Tsugane, S. (2014) Self-Reported Taste Preference Can Be a Proxy for Daily Sodium Intake in Middle-Aged Japanese Adults. Journal of the Academy of Nutrition and Dietetics, 114, 781-787. http://dx.doi.org/10.1016/j.jand.2013.07.043

[19] Frohlich, E.D. and Varagic, J. (2004) The Role of Sodium in Hypertension Is More Complex than Simply Elevating Arterial Pressure. Nature Clinical Practice. Cardiovascular Medicine, 1, 24-30.

[20] Meneton, P., Jeunemaitre, X., de Wardener, H.E. and MacGregor, G.A. (2005) Links between Dietary Salt Intake, Renal Salt Handling, Blood Pressure, and Cardiovascular Diseases. Physiological Reviews, 85, 679-715. http://dx.doi.org/10.1152/physrev.00056.2003

[21] Simon, G. (2003) Experimental Evidence for Blood Pressure-Independent Vascular Effects of High Sodium Diet. American journal of hypertension, 16, 1074-1078.

[22] Leshem, M. (2009) Biobehavior of the Human Love of Salt. Neuroscience \& Biobehavioral Reviews, 33, 1-17. http://dx.doi.org/doi:10.1016/j.neubiorev.2008.07.007

[23] Drewnowski, A. (1997) Taste Preferences and Food Intake. Annual Review of Nutrition, 17, 237-253. http://dx.doi.org/doi:10.1146/annurev.nutr.17.1.237

[24] Birch, L.L. (1999) Development of Food Preferences. Annual Review of Nutrition, 19, 41-62. http://dx.doi.org/doi:10.1146/annurev.nutr.19.1.41

[25] Lampure, A., Schlich, P., Deglaire, A., Castetbon, K., Peneau, S., Hercberg, S. and Mejean, C. (2015) Sociodemographic, Psychological, and Lifestyle Characteristics Are Associated with a Liking for Salty and Sweet Tastes in French Adults. The Journal of Nutrition, 145, 587-594. http://dx.doi.org/doi:10.3945/jn.114.201269

[26] Hudson, J.I., Lalonde, J.K., Coit, C.E., Tsuang, M.T., McElroy, S.L., Crow, S.J., Bulik, C.M., Hudson, M.S., Yanovski, J.A., Rosenthal, N.R. and Pope Jr., H.G. (2010) Longitudinal Study of the Diagnosis of Components of the Metabolic Syndrome in Individuals with Binge-Eating Disorder. The American Journal of Clinical Nutrition, 91, 1568-1573. http://dx.doi.org/doi:10.3945/ajcn.2010.29203

[27] Iso, H., Kobayashi, M., Ishihara, J., Sasaki, S., Okada, K., Kita, Y., Kokubo, Y., Tsugane, S. and Group, J.S. (2006) Intake of Fish and n3 Fatty Acids and Risk of Coronary Heart Disease among Japanese: The Japan Public Health Center-Based (JPHC) Study Cohort I. Circulation, 113, 195-202. http://dx.doi.org/doi:10.1161/CIRCULATIONAHA.105.581355

[28] Kokubo, Y., Iso, H., Ishihara, J., Okada, K., Inoue, M., Tsugane, S. and Group, J.S. (2007) Association of Dietary Intake of Soy, Beans, and Isoflavones with Risk of Cerebral and Myocardial Infarctions in Japanese Populations: The Japan Public Health Center-Based (JPHC) Study Cohort I. Circulation, 116, 2553-2562. http://dx.doi.org/doi:10.1161/CIRCULATIONAHA.106.683755 


\section{Appendix}

The Jichi Medical School Cohort Study Group: Akizumi Tsutsumi (Occupational Health Training Center, University of Occupational and Environmental Health, Fukuoka), Atsushi Hashimoto (Aichi Prefectural Aichi Hospital, Aichi), EijiKajii (Department of Community and Family Medicine, Jichi Medical School, Tochigi), Hideki Miyamoto (former Department of Community and Family Medicine, Jichi Medical School, Tochigi), Hidetaka Akiyoshi (Department of Pediatrics, Fukuoka University School of Medicine), Hiroshi Yanagawa (Saitama Prefectural University, Saitama), Hitoshi Matsuo (Gifu Prefectural Gifu Hospital, Gifu), Jun Hiraoka (Tako Central Hospital, Chiba), Kaname Tsutsumi (Kyushu International University, Fukuoka), Kazunori Kayaba (Saitama Prefectural University), KazuomiKario (Department of Cardiology, Jichi Medical School, Tochigi), Kazuyuki Shimada (Department of Cardiology, Jichi Medical School, Tochigi), Kenichiro Sakai (Akaike Town Hospital, Fukuoka), Kishio Turuda (Takasu National Health Insurance Clinic, Gifu), Machi Sawada (Agawa Osaki National Health Insurance Clinic, Kochi), Makoto Furuse (Department of Radiology, Jichi Medical School, Tochigi), Manabu Yoshimura (Kuze Clinic, Gifu), Masahiko Hosoe (Gero Hot-Spring Hospital, Gifu), Masahiro Igarashi, Masafumi Mizooka (Kamagari National Health Insurance Clinic, Hiroshima), Naoki Nago (Tsukude Health Insurance Clinic, Aichi), Nobuya Kodama (Sakugi Clinic, Hiroshima), Noriko Hayashida (Tako Central Hospital, Chiba), Rika Yamaoka (Awaji-Hokudan Public Clinic, Hyogo), Seishi Yamada (Wara National Health Insurance Hospital, Gifu), Shinichi Muramatsu (Department Neurology, Jichi Medical School, Tochigi), Shinya Hayasaka, Shizukiyo Ishikawa (Department of Community and Family Medicine, Jichi Medical School, Tochigi), Shuzo Takuma (Akaike Town Hospital, Fukuoka), Tadao Gotoh (Wara National Health Insurance Hospital, Gifu), Takafumi Natsume (Oyama Municipal Hospital, Tochigi), Takashi Yamada (Kuze Clinic, Gifu), Takeshi Miyamoto (former Okawa Komatsu National Health Insurance Clinic, Kochi), Tomohiro Deguchi (Akaike Town Hospital, Fukuoka), Tomohiro Saegusa (Sakuma National Health Insurance Hospital, Shizuoka), Yoshihiro Shibano (Saiseikai Iwaizumi Hospital, Iwate) Yoshihisa Ito (Department of Laboratory Medicine, Asahikawa Medical College, Hokkaido), and Yosikazu Nakamura (Department of Public Health, Jichi Medical School).

\section{Abbreviations}

CVD: cardiovascular disease

JMS Cohort Study: Jichi Medical School Cohort Study

HDs: hazardratios

HR-all*: hazard ratios adjusted for age, smoking status and drinking status, history of hyperlipidemia, and education years

HR-all ${ }^{\dagger}$ : hazard ratios adjusted for age, smoking status and drinking status, BMI, HDL-cholesterol, and education years

BMI: body mass index

SBP: systolic blood pressure

DBP: diastolic blood pressure

HDL-C: high density lipoprotein cholesterol

MI: myocardial infarction

SAH: subarachnoid hemorrhage

MONICA Project: Multinational Monitoring of Trends and Determinants in Cardiovascular Disease Project

JACC study: the Japan Collaborative Cohort Study for Evaluation of Cancer Risk 